\title{
Mitochondrial DNA Instability in Cells Lacking Aconitase Correlates with Iron Citrate Toxicity
}

\author{
Muhammad A. Farooq, ${ }^{1}$ Tammy M. Pracheil, ${ }^{1}$ Zhejun Dong, ${ }^{1,2}$ \\ Fei Xiao, ${ }^{2}$ and Zhengchang Liu ${ }^{1}$ \\ ${ }^{1}$ Department of Biological Sciences, University of New Orleans, New Orleans, LA 70148, USA \\ ${ }^{2}$ Key Laboratory of Geriatrics, Beijing Institute of Geriatrics, Beijing Hospital, Ministry of Health, Beijing 100730, China
}

Correspondence should be addressed to Zhengchang Liu; zliu5@uno.edu

Received 15 May 2013; Revised 12 July 2013; Accepted 24 July 2013

Academic Editor: Sergio Giannattasio

Copyright (C) 2013 Muhammad A. Farooq et al. This is an open access article distributed under the Creative Commons Attribution License, which permits unrestricted use, distribution, and reproduction in any medium, provided the original work is properly cited.

\begin{abstract}
Aconitase, the second enzyme of the tricarboxylic acid cycle encoded by ACO1 in the budding yeast Saccharomyces cerevisiae, catalyzes the conversion of citrate to isocitrate. $a c o 1 \Delta$ results in mitochondrial DNA (mtDNA) instability. It has been proposed that Acol binds to mtDNA and mediates its maintenance. Here we propose an alternative mechanism to account for mtDNA loss in aco1 $\Delta$ mutant cells. We found that acol $\Delta$ activated the RTG pathway, resulting in increased expression of genes encoding citrate synthase. By deleting RTG1, RTG3, or genes encoding citrate synthase, mtDNA instability was prevented in acol $1 \Delta$ mutant cells. Increased activity of citrate synthase leads to iron accumulation in the mitochondria. Mutations in MRS3 and MRS4, encoding two mitochondrial iron transporters, also prevented mtDNA loss due to acols. Mitochondria are the main source of superoxide radicals, which are converted to $\mathrm{H}_{2} \mathrm{O}_{2}$ through two superoxide dismutases, Sodl and Sod2. $\mathrm{H}_{2} \mathrm{O}_{2}$ in turn reacts with Fe ${ }^{2+}$ to generate very active hydroxyl radicals. We found that loss of Sod1, but not Sod2, prevents mtDNA loss in acol $\Delta$ mutant cells. We propose that mtDNA loss in acol $\Delta$ mutant cells is caused by the activation of the RTG pathway and subsequent iron citrate accumulation and toxicity.
\end{abstract}

\section{Introduction}

Respiratory metabolism in eukaryotes requires proteins encoded in both the nuclear genome and the mitochondrial genome ( $\mathrm{mtDNA})$. Mitochondrial genomes generally encode a small number of proteins, many of which are involved in respiratory metabolism $[1,2]$. Maintenance of mtDNA is important for cell growth and survival. Oxidative damage to mtDNA causes respiratory deficiency and human diseases [3-5]. In higher eukaryotes, how the mitochondrial genome is maintained and transmitted is not well understood. However, studies using the budding yeast Saccharomyces cerevisiae have generated an abundance of data on how its mitochondrial genome is maintained [6, 7]. Many nuclear-encoded proteins of diverse functions are required for mtDNA maintenance. When mtDNA is mutated, yeast cells form the socalled "petite" mutants. Yeast can be categorized as those with wild type ( $\mathrm{rho}^{+}$cells), extensively deleted (rho ${ }^{-}$petites), or with complete loss of (rho ${ }^{0}$ petites) mtDNA. How mutations in nuclear genes cause defects in mtDNA maintenance is complex and often indirect. For example, mutations in the yeast homolog of frataxin, Yfhl, lead to iron overload in mitochondria, defects in maturation of proteins containing iron-sulfur clusters such as the TCA cycle enzyme aconitase, mtDNA instability and respiratory deficiency, and so forth [8-10]. Yeast studies have suggested that iron citrate toxicity may be responsible for $y f h 1$ mutant phenotypes $[11,12]$. Respiratory metabolism generates reactive oxygen species such as superoxide radicals. Superoxide dismutases, Sod1 and Sod2, localized in the cytoplasm and mitochondria, respectively, are responsible for converting superoxide radicals to relatively harmless hydrogen peroxide [13], which can react with ferrous iron $\left(\mathrm{Fe}^{2+}\right)$ to generate highly reactive hydroxyl radicals through the Fenton reaction. Hydrogen peroxide is detoxified by enzymes such as catalases, converting hydrogen peroxide to oxygen and water [14]. Mutations in yeast catalases and 
superoxide dismutase lead to oxidative damage and reduced resistance to oxidants [14-16].

Besides resulting in respiratory deficiency, mutations in TCA cycle enzyme encoding genes also lead to variable defects in mtDNA maintenance $[17,18]$. The most severe phenotype is caused by mutations in the ACO1 gene encoding aconitase, followed by the IDH1 gene encoding a subunit of mitochondrial isocitrate dehydrogenase [19]. It has been proposed that Acol has a novel function in mediating mtDNA maintenance by directly binding mtDNA $[20,21]$. Mutations in ACO1 and IDH1 share several growth defect phenotypes, which can be partially rescued by mutations in CIT1, encoding the mitochondrial isoform of citrate synthase [17]. Expression of CIT1, ACO1, IDH1, and IDH2 is under dual control of two transcriptional regulatory complexes, Rtg1/3 and Hap2/3/4/5 [22]. In cells with reduced or defective respiratory functions, expression of these genes is under increased control of Rtg1/3. Rtg1 and Rtg3 are two basic helix-loophelix transcription factors in the retrograde response pathway that mediates signaling from mitochondria to the nucleus [23]. Activation of $\mathrm{Rtg} 1 / 3$ requires a cytoplasmic protein, Rtg2, which has an N-terminal ATP binding domain in the Hsp70/actin/sugar kinase ATP binding domain superfamily [24]. The retrograde response pathway, also known as the RTG pathway, is activated in response to defects in mitochondrial respiratory function. Cit1, Acol, and Idh1/2 promote synthesis of $\alpha$-ketoglutarate, a precursor of glutamate, which is a potent repressor of the RTG pathway [23]. Mutations in $A C O 1$, which lead to both a block in mitochondrial respiratory function and glutamate auxotrophy [25-27], therefore, likely activate the RTG pathway. However, it is not clear whether the RTG pathway contributes to the phenotypes of acol mutants.

Mutations in RTG2 and CIT2 have been reported to suppress mtDNA instability due to mutations in YFH1 [12]. In this study, we provide an alternative model to account for mtDNA loss due to an acol $\Delta$ mutation. We found that mutations in either RTG genes, genes encoding citrate synthases, genes encoding mitochondrial iron transporters, or SOD1 suppress acol $\Delta$-induced mtDNA loss. Therefore, we propose that iron citrate toxicity contributes to $a c o 1 \Delta$ mutant phenotypes.

\section{Materials and Methods}

\subsection{Strains, Plasmids, Growth Media, and Growth Conditions.} Yeast strains and plasmids used in this study are listed in Tables 1 and 2, respectively. Yeast mutant strains were created by either direct transformation with gene knockout cassettes or through meiotic segregation analysis of heterozygous diploids. Mutations were confirmed by PCRgenotyping, standard genotyping based on selection markers, phenotypic analysis, and/or immunoblotting using antibody against Acol. The BY4741 rho ${ }^{0}$ strain was generated by one passage of rho $^{+}$cells grown in YPD medium supplemented with $15 \mu \mathrm{g} / \mathrm{mL}$ ethidium bromide. Yeast cells were grown in SD $(0.67 \%$ yeast nitrogen base plus $2 \%$ dextrose), YNBCasD (SD medium plus $1 \%$ casamino acids), YNBcasR ( $0.67 \%$ yeast nitrogen base, $1 \%$ casamino acids, and $2 \%$ raffinose), YPD (1\% yeast extract, $2 \%$ peptone, $2 \%$ dextrose), or YPEthanol ( $1 \%$ yeast extract, $2 \%$ peptone, $2 \%$ ethanol) medium at $30^{\circ} \mathrm{C}$. When necessary, amino acids, adenine, and/or uracil were added to the growth medium at standard concentrations to cover auxotrophic requirements [28].

2.2. Yeast Transformation and $\beta$-Galactosidase Activity Assays. Plasmids were transformed into yeast strains using the highefficiency lithium acetate-PEG method and $\beta$-galactosidase assays were carried out as described [28]. For each plasmid and strain combination, assays were conducted in duplicates, and independent experiments were carried out two times. Specific activity of $\beta$-galactosidase is expressed as nmols of $o$-nitrophenol generated from substrate $o$-nitrophenyl- $\beta$-Dgalactoside per min per mg protein.

2.3. DAPI Staining of Nuclear and Mitochondrial DNA and FLuorescence Microscopy. DAPI (4'6,-diamidino-2-phenylindole) staining of nuclear and mitochondrial DNA was carried out as described [28]. Briefly, yeast strains were grown in liquid YPD or YNBcasD medium at $30^{\circ} \mathrm{C}$ overnight to $A_{600} \sim 0.8$. Cells were collected by centrifugation and treated with $1 \mu \mathrm{g} / \mathrm{mL}$ DAPI in $95 \%$ ethanol for $30 \mathrm{~min}$ and cell pellets were washed with sterile water three times. DAPI-stained DNA molecules in fixed cells were observed by fluorescence microscopy using a Nikon Eclipse E800 microscope equipped with an HBO 100 W/2 mercury arc lamp, a Nikon Plan Fluor 100X objective lens, and epifluorescence with a Nikon UV2E/C filter set (excitation 340-380 nm and emission 435$485 \mathrm{~nm}$ ). Digital images were acquired with Photometrics Coolsnap fx CCD camera and Metamorph Imaging Software (Molecular Devices, Sunnyvale, CA) and processed using ImageJ (National Institutes of Health) and Adobe Photoshop (Mountain View, CA).

2.4. Citrate Analysis. Cells were grown in $10 \mathrm{~mL}$ YPD medium overnight to $\sim \mathrm{OD}_{600}$ 1.0. Cultures were chilled in ice-cold water for $22 \mathrm{~min}$ and cells were collected by centrifugation at $4^{\circ} \mathrm{C}$. Cell pellets were then washed twice in chilled water. Citrate levels were determined using a citrate assay kit (BioVision, CA, USA). Cells were disrupted in $500 \mu \mathrm{L}$ Assay Buffer in the kit using glass beads method. Cell extract was clarified by centrifugation at $21,000 \mathrm{~g}$ at $4^{\circ} \mathrm{C}$ for $15 \mathrm{~min} .20 \mathrm{uL}$ cell extract was analyzed for protein concentration using Bradford assay and the rest of cell extract ( 350) was deproteinized in Amicon Ultra 4 column (10 kDa cutoff). Deproteinized extract was analyzed for citrate levels according to the protocol provided by the manufacturer. Citrate levels in different strains were normalized by protein concentration of cellular extract prior to deproteinization. Citrate concentration in the wild-type strain was $7.97 \mathrm{nmols} / \mathrm{mg}$ proteins, which was arbitrarily set as 1 unit. Citrate concentration in the wild-type strain determined in this study is similar to $\sim 1.1 \mathrm{nmols} / 10^{7}$ cells reported previously [12]. 
TABLE 1: Strains used in this study.

\begin{tabular}{|c|c|c|}
\hline Strain & Genotype & Source \\
\hline BY4741 & MATa ura3 leu2 his3 met15 & Research genetics \\
\hline BY4731 & MATa ura3 leu 2 met 15 & Research genetics \\
\hline BY 4742 & MATa ura3 leu2 his3 lys2 & Research genetics \\
\hline $\mathrm{BY} 4741\left(\mathrm{rho}^{0}\right)$ & BY4741 rho $^{0}$ derivative & This study \\
\hline ZLY2630 & BY4741 aco1::kanMX4 & This study \\
\hline ZLY1264 & BY4741 rtg1::kanMX4 & Research genetics \\
\hline ZLY1267 & BY4741 rtg2::kanMX4 & Research genetics \\
\hline ZLY1273 & BY4741 rtg3::kanMX4 & Research genetics \\
\hline ZLY2568 & BY4742 rtg1::LEU2 & This study \\
\hline ZLY143 & BY4731 rtg2::LEU2 & This study \\
\hline ZLY2570 & BY4742 rtg3::URA3 & This study \\
\hline ZLY2648 & BY4741 rtg1::LEU2 aco1::kanMX4 & This study \\
\hline ZLY2631 & BY4741 rtg2::LEU2 aco1::kanMX4 & This study \\
\hline ZLY2652 & BY4741 rtg3::URA3 aco1::kanMX4 & This study \\
\hline ZLY3206 & 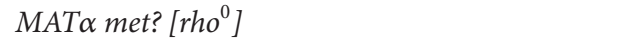 & This study \\
\hline CS725-3A & MATa ura3 leu2 his3 met15 cit1/2/3::kanMX4 & {$[21]$} \\
\hline ZLY2545 & CS725-3A aco1::HIS3 & {$[21]$} \\
\hline RBY353 & BY4741 cit1::kanMX4 & Research genetics \\
\hline RBY355 & BY4741 cit2::kanMX4 & Research genetics \\
\hline RBY356 & BY4741 cit3::kanMX4 & Research genetics \\
\hline ZLY2603 & MATa ura3 leu2 met15 aco1::kanMX4 & This study \\
\hline RBY469 & BY4741 cit1::kanMX4 aco1::kanMX4 & This study \\
\hline RBY277 & BY4741 cit2::kanMX4 aco1::kanMX4 & This study \\
\hline RBY363 & BY4741 cit3::kanMX4 aco1::kanMX4 & This study \\
\hline ZLY854 & BY4741 cit1/2::kanMX4 aco1::kanMX4 & This study \\
\hline BY4741 (mrs3 mrs4) & BY4741 mrs3::kanMX4 mrs4::kanMX4 & {$[29]$} \\
\hline ZLY3505 & BY4741 mrs3::kanMX4 mrs4::kanMX4 aco1::HIS3 & This study \\
\hline ZLY4603 & BY4741 sod1::kanMX4 & This study \\
\hline BY4741 (sod2) & BY4741 sod2::kanMX4 & Research genetics \\
\hline TPY1507 & BY4741 sod1::kanMX4 aco1::kanMX4 & This study \\
\hline ZLY3973 & BY4741 sod2::kanMX4 aco1::kanMX4 & This study \\
\hline
\end{tabular}

TABLE 2: Plasmids used in this study.

\begin{tabular}{lll}
\hline Plasmid & Description & Source \\
\hline pRS416 & A yeast centromeric plasmid carrying URA3 selection marker & [30] \\
pUC-rtg1::LEU2 & An $r$ tg1::LEU2 disruption cassette in pUC19 & [31] \\
pUC-rtg2::LEU2 & An $r$ tg2::LEU2 disruption cassette in pUC19 & [31] \\
pUC-rtg3::URA3 & An $r$ tg3::URA3 disruption cassette in pUC19 & {$[32]$} \\
pBS-aco1::HIS3 & An aco1::HIS3disruption cassette in pBluescript & This study \\
pCIT2-lacZ & A CIT2-lacZ reporter gene on the plasmid pWCJ (CEN URA3) & This study \\
pRS303-SOD1 & The SOD1 gene was cloned into the integrative plasmid pRS303 & This study \\
\hline
\end{tabular}

\section{Results and Discussion}

3.1. acolD Activates the RTG Pathway. Mutations in ACO1 lead to both respiratory deficiency and glutamate starvation, which are expected to activate the RTG pathway. To test this possibility, we determined the effect of an acol $\Delta$ mutation on the expression of a CIT2-lacZ reporter gene, which has been used extensively as a readout of the activity of the RTG pathway [22, 24, 33-35]. Expression of CIT2-lacZ was assessed in wild-type rho ${ }^{+}$and acol $\Delta$ mutant cells using $\beta$-galactosidase assays. Since acol $\Delta$ cells are rho ${ }^{0}$ petites, we also determined CIT2-lacZ expression in otherwise wild-type rho ${ }^{0}$ cells. Cells were grown in rich media with either raffinose or dextrose (D-glucose) as the sole carbon source, which have been used in studies on the RTG pathway and mitochondrial genome maintenance, respectively $[12,20,21,36]$. In cells grown in raffinose medium, CIT2-lacZ expression was 4 -fold higher in rho $^{0}$ cells compared to rho ${ }^{+}$cells, consistent with previous 


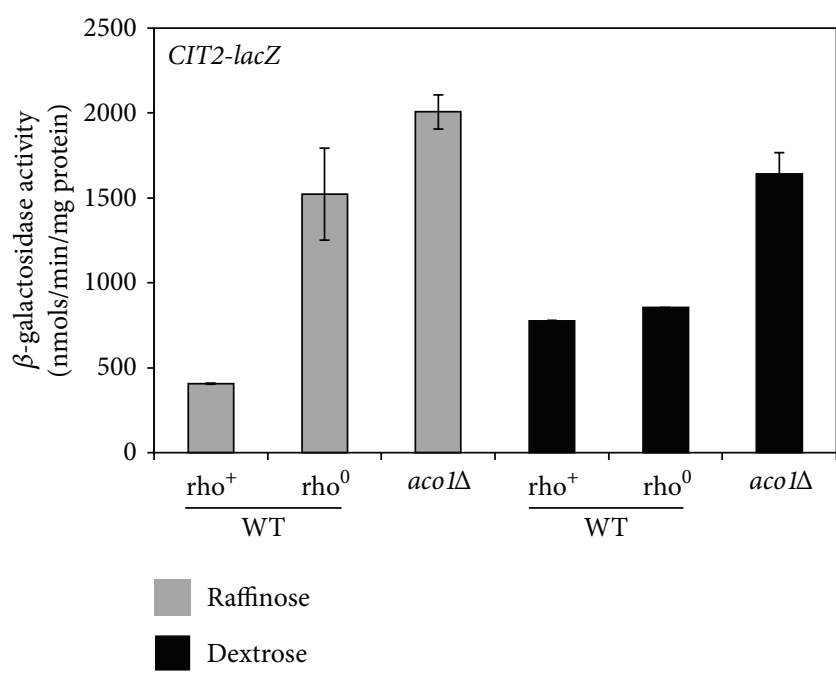

FIGURE 1: The RTG pathway is activated in aco1 $\Delta$ mutant cells grown in dextrose medium. Wild-type rho $^{+}$(WT, BY4741), its rho derivative, and aco1s (ZLY2603) mutant strains were transformed with a centromeric plasmid encoding a CIT2-lacZ reporter gene (pCIT2lacZ) and transformants were grown in YNBcasR (Raffinose) and YNBcasD (Dextrose) medium to mid-logarithmic phase. Cells were collected and $\beta$-galactosidase assays were conducted as described in Section 2.

reports that the RTG pathway is activated in rho ${ }^{0}$ cells $[33,36]$. An aco1s mutation induced CIT2-lacZ expression slightly higher than in wild-type rho cells (Figure 1). In cells grown in dextrose medium, CIT2-lac $Z$ expression in rho ${ }^{0}$ cells is only marginally higher than in $\mathrm{rho}^{+}$cells, whereas an acols mutation almost doubled CIT2-lacZ expression. Activation of the RTG pathway is strain dependent [37]. The lack of induction of the RTG pathway due to loss of mtDNA in dextrose-grown BY4741 background strains used in this study can be partly attributed to a doubling of CIT2-lacZ expression in rho ${ }^{+}$cells grown in this medium compared to raffinose medium, which is consistent with activation of the RTG pathway due to compromised mitochondrial respiratory function since dextrose suppresses respiratory metabolism in yeast. Altogether, these data indicate that an acol $\Delta$ mutation leads to activation of the RTG pathway.

3.2. Mutations in RTG Genes Prevent mtDNA Loss due to aco1 $\Delta$. To determine whether activation of the RTG pathway in acol $\Delta$ mutant cells contributes to mtDNA loss, rtg1 aco $1 \Delta, r \operatorname{tg} 2 \Delta a \operatorname{co} 1 \Delta$, and $r \operatorname{tg} 3 \Delta$ aco1 $\Delta$ double mutants were constructed and examined for the presence or absence of mtDNA. These double mutant strains were created by crossing respective haploid mutant strains to form heterozygous diploid mutants, which were then sporulated to generate desired haploid segregants. Seven $\operatorname{rtg} 1 \Delta$ acols, seven $\operatorname{rtg} 2 \Delta$ acol $\Delta$, and $\operatorname{six} \operatorname{rtg} 3 \Delta$ acol $\Delta$ double mutant segregants were obtained. Eight acols single mutant segregants were also isolated similarly. DAPI, a DNA-specific probe that forms a fluorescent complex [38], was then used to visualize mtDNA using fluorescence microscopy in these mutants along with wild-type rho $^{+}$and rho $^{0}$ strains. In addition to nuclear DNA, DAPI staining revealed punctate cytoplasmic structures of mtDNA in wild-type rho $^{+}$cells grown in YPD medium (Figure 2(a)) [38]. In contrast, mtDNA was absent in both wild-type rho ${ }^{0}$ and acol $\Delta$ mutant cells, consistent with previous reports that Acol is required for mtDNA maintenance $[17,18,21]$. Interestingly, mtDNA was maintained in rtg1 $\Delta$ $a c o 1 \Delta, r \operatorname{tg} 2 \Delta a c o 1 \Delta$, and $r \operatorname{tg} 3 \Delta$ aco $1 \Delta$ double mutant strains, indicating that Rtg proteins mediate mtDNA instability in aco1s mutant cells. The percentage of rho ${ }^{0}$ cells was quantified from DAPI-stained images and a large majority of $r \operatorname{tg} \Delta$ acols double mutant cells were found to contain mtDNA (Figure 2(b)). Among seven $r \operatorname{tg} 2 \Delta$ acol $\Delta$ double mutant segregants from heterozygous $\mathrm{rtg} 2 \Delta / R T G 2$ acol $\Delta / A C O 1$ diploid mutant cells, all were found to maintain mtDNA. Similarly, all of the seven $r \operatorname{tg} 1 \Delta$ acol and $\operatorname{six} r \operatorname{tg} 3 \Delta$ acol $\Delta$ double mutant segregants were also found to be rho ${ }^{+}$cells. In contrast, all of the eight acol $\Delta$ single mutant segregants from a heterozygous aco1 $\triangle / A C O 1$ diploid mutant have lost mtDNA. Together, our data suggest that mtDNA instability in acols mutant cells may result from activation of the RTG pathway.

Damages to mtDNA can lead to extensive deletions (rho ${ }^{-}$) or point mutations $\left(\right.$mit $\left.^{-}\right)[6,7]$. Yeast strains that carry these two types of mutant mitochondrial genomes are respiratory deficient. To determine whether the mtDNA in the $r \operatorname{tg} \Delta$ acol $\Delta$ double mutant strains is functional, we conducted a complementation assay by crossing wild-type rho $^{+}$, wild-type $\mathrm{rho}^{0}, r \operatorname{tg} 1 \Delta, r \operatorname{tg} 2 \Delta, r \operatorname{tg} 3 \Delta, r \operatorname{tg} 1 \Delta \operatorname{aco} 1 \Delta, r \operatorname{tg} 2 \Delta$ aco $1 \Delta$, and $r \operatorname{tg} 3 \Delta$ aco $1 \Delta$ mutant strains to a rho ${ }^{0}$ tester strain of opposite mating type with wild-type nuclear ACO1 gene and analyzing the respiratory capacity of the resultant diploid strains. $r \operatorname{tg} 1 \Delta a c o 1 \Delta, r \operatorname{tg} 2 \Delta a c o 1 \Delta$, and $r \operatorname{tg} 3 \Delta$ aco $1 \Delta$ mutant strains were unable to utilize carbon sources that require respiratory metabolism such as ethanol because they are defective in the TCA cycle (data not shown), and Figure 2(c) shows that diploids generated from crossing wild-type rho ${ }^{+}$, $r \operatorname{tg} 1 \Delta, r \operatorname{tg} 2 \Delta$, and $r \operatorname{tg} 3 \Delta$ strains with the rho ${ }^{0}$ tester strain were able to grow on ethanol medium. In contrast, diploids from crosses involving wild-type rho ${ }^{0}$ or the acols single mutant were unable to grow on ethanol medium, consistent with the absence of mtDNA in these diploids. Remarkably, diploids generated from the rho ${ }^{0}$ tester strain and $r \operatorname{tg} \Delta$ acol $\Delta$ double mutants were able to grow on ethanol medium, indicating that mtDNA in $r \operatorname{tg} \Delta$ acol $\Delta$ cells are functional.

3.3. Mutations in Genes Encoding Citrate Synthase, Primarily CIT1, Prevent mtDNA Loss due to acold. What is the mechanism of acol $\Delta$ suppression by mutations in RTG genes? The $R T G$ pathway is required for glutamate biosynthesis in cells with reduced respiratory function by regulating expression of CIT1, CIT2, ACO1, IDH1, and IDH2 [23]. It has been shown previously that a cit $1 \Delta$ mutation partially suppresses mtDNA loss in aco1s mutant cells and that mutations in CIT2 and RTG2 rescue respiratory deficiency in $y f h 1 \Delta$ mutant cells $[12,17]$. Three genes, CIT1, CIT2, and CIT3, encode citrate synthase in yeast, with CIT1 and CIT3 encoding the two mitochondrial isoforms and CIT2 encoding the peroxisomal isoform [39-41]. Therefore, suppression of mtDNA loss in 


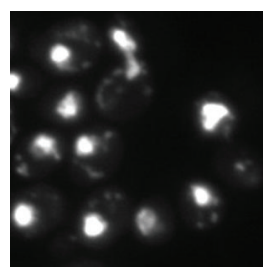

WT $\left(\right.$ rho $\left.^{+}\right)$

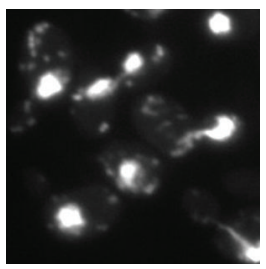

$\operatorname{rtg} 1 \Delta \operatorname{aco} 1 \Delta$

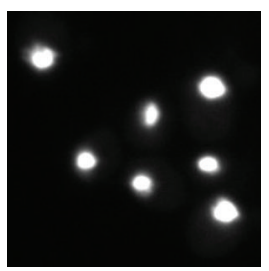

$\mathrm{WT}\left(\mathrm{rho}^{0}\right)$

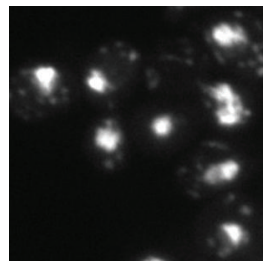

rtg $2 \Delta \operatorname{aco} 1 \Delta$

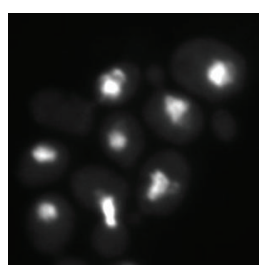

acols

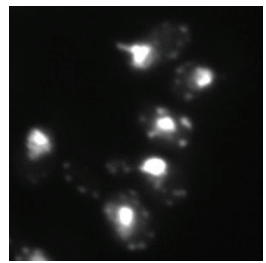

$\operatorname{rtg} 3 \Delta \operatorname{aco} 1 \Delta$

(a)

\begin{tabular}{|c|c|c|}
\hline Strains & rho $^{0}$ cells (\%) & $\begin{array}{l}\text { Number of } \\
\text { cells analyzed }\end{array}$ \\
\hline $\mathrm{WT}\left(\mathrm{rho}^{+}\right)$ & $2.5 \%$ & 551 \\
\hline $\mathrm{WT}\left(\mathrm{rho}^{0}\right)$ & $100.0 \%$ & 433 \\
\hline $\operatorname{acol} 1 \Delta$ & $100.0 \%$ & 440 \\
\hline $\operatorname{rtg} 1 \Delta \operatorname{aco} 1 \Delta$ & $4.7 \%$ & 406 \\
\hline $\operatorname{rtg} 2 \Delta \operatorname{aco} 1 \Delta$ & $18.3 \%$ & 454 \\
\hline $\operatorname{rtg} 3 \Delta a \cos \Delta$ & $6.0 \%$ & 399 \\
\hline
\end{tabular}

(b)

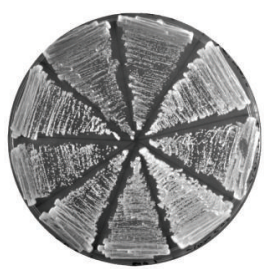

Dextrose

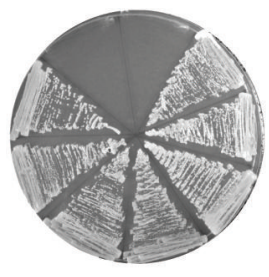

Ethano

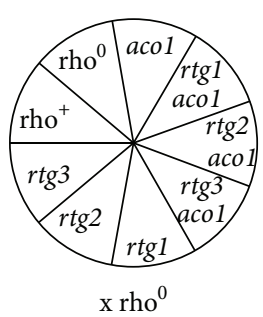

(c)

FIGURE 2: mtDNA is maintained in $r \operatorname{tg} 1 \Delta$ acol, $r \operatorname{tg} 2 \Delta$ acols, and $r \operatorname{tg} 3 \Delta$ acol $\Delta$ mutant cells. (a) Cells grown exponentially in YPD medium were stained with DAPI and then examined using fluorescence microscopy. WT ( rho $^{+}$), BY4741; WT (rho ${ }^{0}$ ), BY4741 rho ${ }^{0}$; acols, ZLY2630; rtg1 $\Delta$ aco1 $\Delta$, ZLY2648; rtg2 $\Delta$ aco1 $\Delta$, ZLY2631; rtg3 $\Delta$ aco1 $\Delta$, ZLY2652. (b) Quantitative analysis of the percentage of rho ${ }^{0}$ cells in yeast strains based on DAPI-staining images. (c) mtDNA in $r \operatorname{tg} \Delta$ aco1 $\Delta$ double mutant cells is functional. Strains as described in (a) as well as $r t g 1 \Delta$, $r t g 2 \Delta$, and $r$ tg $3 \Delta$ single mutants were crossed to a rho ${ }^{0}$ tester strain $\left(\mathrm{X} \mathrm{rho}^{0}\right)$ of opposite mating type. Diploids were selected and grown on YPD (Dextrose) and YPEthanol (Ethanol) medium. Pictures of cells were taken after 3 days' growth at $30^{\circ} \mathrm{C}$.

rtg1 $\operatorname{aco} 1 \Delta, \operatorname{rtg} 2 \Delta a c o 1 \Delta$, and $r \operatorname{tg} 3 \Delta$ aco1 $\Delta$ double mutant cells may be due to reduced expression of genes encoding citrate synthase. To confirm this possibility, we introduced an acol $\Delta$ mutation into a cit $1 \Delta$ cit $2 \Delta$ cit $3 \Delta$ triple mutant in the BY4741 strain background generated by Chen et al. [21]. The presence or absence of mtDNA in the resultant quadruple mutant cells was examined by DAPI staining and fluorescence microscopy. Figure 3(a) shows that the cit $1 \Delta$ cit $2 \Delta$ cit $3 \Delta$ acol $\Delta$ quadruple mutant maintained mtDNA. To determine which citrate synthase-encoding gene(s) is responsible for mtDNA loss in acol $\Delta$ mutant cells, we generated cit1 $\Delta$ acol $\Delta$, cit $2 \Delta$ aco1 $\Delta$, and cit $3 \Delta$ acol $\Delta$ double mutants, as well as an aco $1 \Delta$ cit $1 \Delta$ cit $2 \Delta$ triple mutant by crossing respective haploid mutant strains followed by meiotic segregation analysis. Using DAPI staining and fluorescence microscopy, we found that six out of eight cit1 $\Delta$ acol $\Delta$ double mutants, zero out of six cit $2 \Delta$ acol $\Delta$ double mutants, zero out of six cit $3 \Delta$ acol $\Delta$ double mutants, and six out of $\operatorname{six}$ cit $1 \Delta$ cit $2 \Delta$ aco $1 \Delta$ triple mutant strains maintained mtDNA (Figure 3 and data not shown). We also calculated the percentage of rho cells in a cit $1 \Delta$ aco1 $\Delta$ double, a cit $1 \Delta$ cit $2 \Delta$ acol $\Delta$ triple, and a cit $1 \Delta$ cit $2 \Delta$ cit $3 \Delta$ acol $\Delta$ quadruple mutant and found that over $90 \%$ 


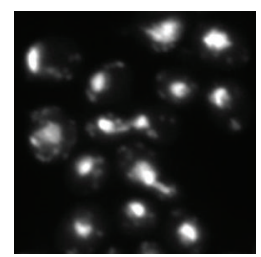

WT $\left(\right.$ rho $\left.^{+}\right)$

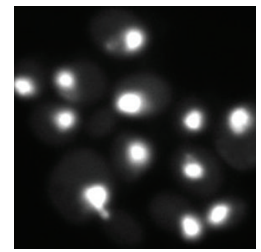

$\operatorname{cit} 2 \Delta \operatorname{aco} 1 \Delta$

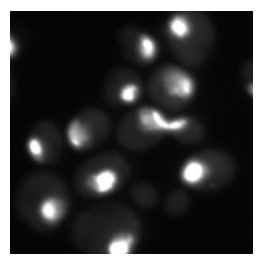

$\operatorname{acol} 1 \Delta$

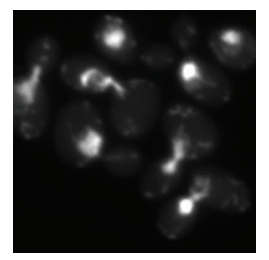

$\operatorname{cit} 1 \Delta \operatorname{cit} 2 \Delta$ aco $1 \Delta$

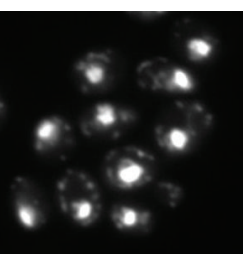

$\operatorname{cit} 1 \Delta$ acols

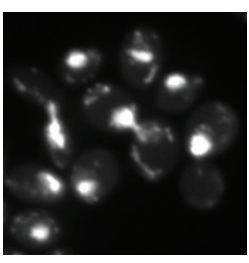

cit $1 \Delta \operatorname{cit} 2 \Delta \operatorname{cit} 3 \Delta$ aco $1 \Delta$

(a)

\begin{tabular}{ccc} 
Strains & rho $^{0}$ cells (\%) & $\begin{array}{c}\text { Number of } \\
\text { cells analyzed }\end{array}$ \\
\hline aco $1 \Delta$ & $100.0 \%$ & 440 \\
cit $1 \Delta$ aco $1 \Delta$ & $6.5 \%$ & 432 \\
cit1 $\operatorname{cit} 2 \Delta$ aco $1 \Delta$ & $9.9 \%$ & 444 \\
cit1 $\operatorname{cit} 2 \Delta$ cit $3 \Delta$ aco $1 \Delta$ & $8.2 \%$ & 454 \\
\hline
\end{tabular}

(b)

FIGURE 3: Mutations in genes encoding citrate synthase prevent mtDNA loss due to acol $\Delta$. (a) Cells grown in YPD medium to mid-logarithmic phase were stained with DAPI and examined using fluorescence microscopy. WT (rho ${ }^{+}$), BY4741; aco1 $\Delta$, ZLY2630; cit1 $a$ acol $\Delta$, RBY469; cit2 $\Delta$

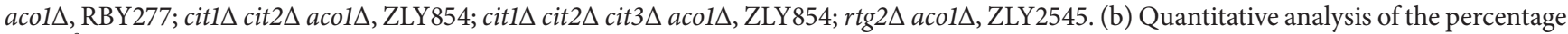
of rho ${ }^{0}$ cells in yeast strains based on DAPI-staining images.

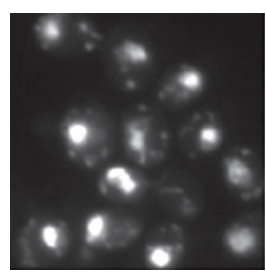

$\mathrm{WT}\left(\mathrm{rho}^{+}\right)$

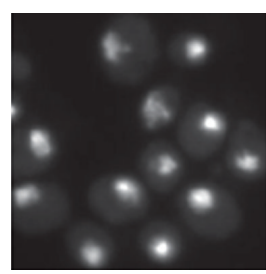

aco $1 \Delta$

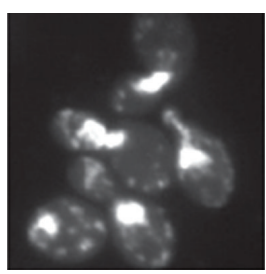

$m r s 3 \Delta m r s 4 \Delta a c o 1 \Delta$

FIGURE 4: Mutations in MRS3 and MRS4, encoding mitochondrial iron transporters, prevent mtDNA loss due to acol $\Delta$. Cells grown in YPD medium to mid-logarithmic phase were stained with DAPI and examined using fluorescence microscopy. WT $\left(\mathrm{rho}^{+}\right), \mathrm{BY} 4741$; aco1s, ZLY2630; $m r s 3 \Delta$ mrs4 $\Delta$ aco1s, ZLY3505.

cells from these mutants maintained mtDNA (Figure 3(b)). Taken together, these data suggest that citrate synthase is the target of the RTG pathway that mediates mtDNA instability in aco1s mutant cells and that Citl is primarily responsible for this phenotype in the BY4741 strain background.

\subsection{Mutations in Genes Encoding Mitochondrial Iron Trans-} porters Mrs3 and Mrs4 Prevent mtDNA Loss due to acols. It has been proposed that iron citrate toxicity contributes to oxidative damage and mtDNA loss in $y f h 1 \Delta$ mutant cells, which have higher levels of cellular and mitochondrial iron $[8,12]$. Mutations in RTG2 and CIT2 reduce petite formation

in $y f h 1 \Delta$ mutants by lowering cellular citrate and iron levels. Suppression of mtDNA loss in acols mutant cells by mutations in $R T G$ genes and genes encoding citrate synthase prompted us to test whether mitochondrial iron overload is responsible for mtDNA instability in acols mutants. Mitochondrial iron transport is mediated by iron transporters Mrs3 and Mrs4 [11, 29, 42, 43], mutations of which rescue mtDNA loss in $y f h 1 \Delta$ mutants. To this end, we generated an $m r s 3 \Delta m r s 4 \Delta$ acol $\Delta$ triple mutant by introducing an acol $\Delta$ mutation into an $m r s 3 \Delta m r s 4 \Delta$ double mutant. DAPI staining of the triple mutant showed that mtDNA was maintained (Figure 4). Furthermore, quantitative analysis showed that the percentage of rho ${ }^{0}$ cells in the triple mutant was $0.4 \%$, slightly lower than $2.5 \%$ in wild-type $\mathrm{rho}^{+}$cells. The $m r s 3 \Delta$ $m r s 4 \Delta$ acols triple mutant was also mated to a rho ${ }^{0}$ tester strain and the resulting diploids were streaked onto plates with ethanol as the sole carbon source. We found that the diploids could grow on ethanol medium, indicating that mtDNA in the $m r s 3 \Delta m r s 4 \Delta$ acol $\Delta$ triple mutant is functional (data not shown). Together, this data supports the notion that mtDNA loss in aco1s mutant cells is due to iron citrate toxicity.

It has been reported that the supplementation of exogenous iron $\left(1 \mathrm{mM} \mathrm{FeSO}_{4}\right)$ or raising $\mathrm{pH}$ of the growth medium reduces petite frequency in acol $\Delta$ mutant cells grown in YPGalactose medium [17]. Thus, Lin et al. proposed that some of the effects of elevated citrate levels in aco1s are 


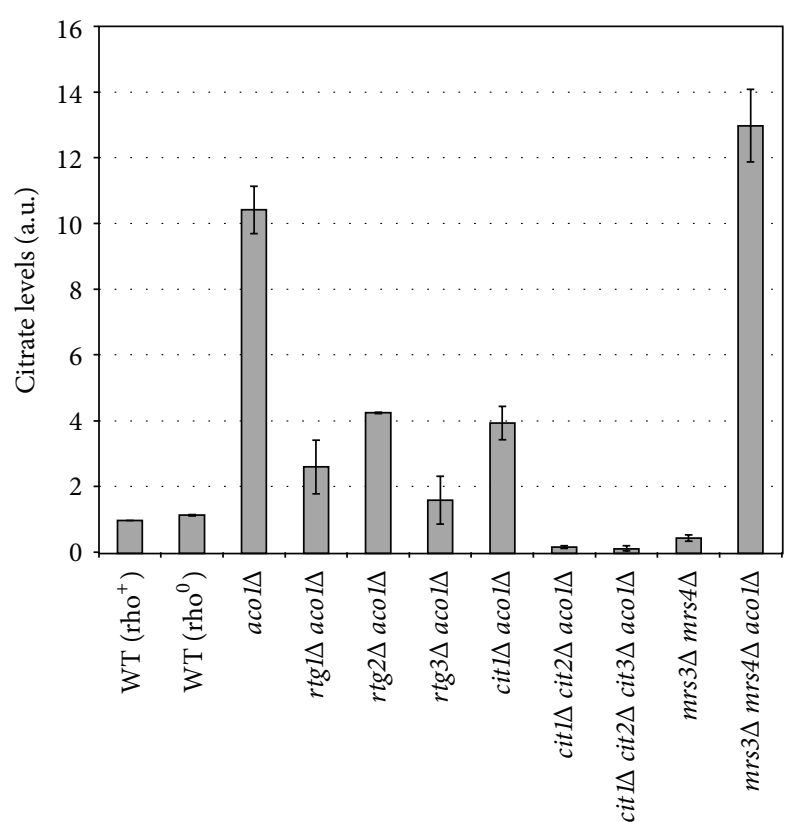

FIGURE 5: Citrate levels in wild-type (WT) and indicated mutant strains. Citrate concentration was determined as described in Section 2. The results are the average of two independent experiments.

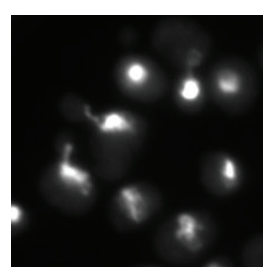

$\operatorname{acol\Delta }$

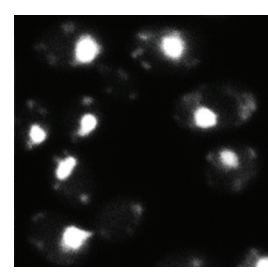

$\operatorname{sod} 1 \Delta \operatorname{aco} 1 \Delta$

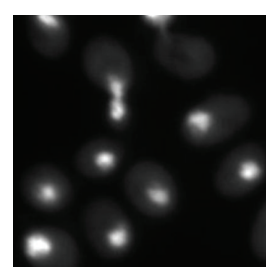

$\operatorname{sod} 2 \Delta \operatorname{aco} 1 \Delta$
FIgURE 6: A mutation in SOD1, but not SOD2, suppresses mtDNA loss due to aco1 $\Delta$. Cells grown in YPD medium to mid-logarithmic phase were stained with DAPI and examined using fluorescence

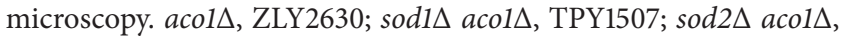
ZLY3973.

due to the ionized form of this metabolite rather than to the formation of a citrate/iron chelate that could produce oxidative damage to mtDNA. These observations seem to contradict our conclusion. However, iron homeostasis is a highly complicated process [44]. It was not established that exogenous iron actually increased iron levels in the mitochondria in Lin et al.s study. It is also possible that growth conditions (YPD in this study versus YPGalactose in Lin et al's study) and/or strain backgrounds (BY4741 versus W303-1B) may affect mtDNA loss associated with aco1s mutations.

3.5. Iron Citrate Toxicity Correlates with mtDNA Loss in acol $1 \mathrm{Mutant}$ Cells. To affirm our hypothesis that iron citrate toxicity contributes to mtDNA loss in acols mutant cells, we determined citrate levels in wild-type and various mutant strains and found that acols increased the citrate level by 10.5-fold (Figure 5), which is consistent with an 11.9-fold increase reported by Lin et al. [17]. This increase is not due to that acols mutants are rho ${ }^{0}$ cells since the citrate level in wild-type rho cells was only $16 \%$ higher than that of wild-type rho ${ }^{+}$cells. Mutations in RTG1, RTG2, and RTG3 reduced citrate levels in acol $\Delta$ background cells by $59-85 \%$. Similarly, cit $1 \Delta$ reduced citrate concentration by $62 \%$ in acol $\Delta$ background cells, which is comparable to an $89 \%$ decrease reported by Lin et al. A double mutation in CIT1 and CIT2 reduced citrate concentration by $98 \%$ in acol $\Delta$ background cells, and an additional mutation in CIT3 did not significantly further reduce citrate levels. Clearly, there is strong correlation between the suppression of mtDNA loss phenotype and lower citrate levels in acol $\Delta$ background strains. We also determined the effect of an $m r s 3 \Delta$ mrs $4 \Delta$ double mutation on citrate levels. In comparison to a wild-type rho $^{+}$strain, citrate concentration in an $m r s 3 \Delta m r s 4 \Delta$ double mutant was $53 \%$ lower. In contrast, citrate concentration in an $m r s 3 \Delta$ $m r s 4 \Delta$ acol $\Delta$ triple mutant is $24 \%$ higher than that of an acol $\Delta$ mutant. Since $m r s 3 \Delta m r s 4 \Delta$ aco $1 \Delta$ mutant cells maintained mtDNA, we propose that high levels of citrate per se are not sufficient to lead to mtDNA loss and that citrate toxicity requires certain levels of iron in the mitochondria. Together with our genetic data, these biochemical results suggest that iron citrate toxicity accounts for $\mathrm{mtDNA}$ loss in acol $\Delta$ mutant cells.

3.6. A Mutation in SOD1, but Not SOD2, Prevents mtDNA Loss due to aco1 $\Delta$. Our data suggest that high levels of citrate cause mtDNA instability in acol $\Delta$ mutant cells likely due to iron citrate toxicity. Iron reacts with hydrogen peroxide in the Fenton reaction to produce highly active, potent hydroxyl radicals, which cause oxidative damage to mitochondria. Hydrogen peroxide is partly produced by the superoxide dismutases, Sod1 in the cytoplasm and Sod2 in the mitochondrial matrix [12]. We hypothesized that a reduced production of hydrogen peroxide due to mutations in SOD1 or SOD2 might suppress mtDNA loss in aco1 $\triangle$ mutant cells by lowering the amount of hydroxyl radicals produced via the Fenton reaction. Therefore, we generated $\operatorname{sod} 1 \Delta$ acol $\Delta$ and $\operatorname{sod} 2 \Delta$ acol $\Delta$ double mutants by crossing respective haploid mutant strains followed by meiotic segregation analysis. The resultant double mutant strains were analyzed for mtDNA presence by DAPI staining and fluorescence microscopy. 33 out of $34 \operatorname{sod} 1 \Delta$ acols double mutant strains generated maintained mtDNA while 6 out of $6 \operatorname{sod} 2 \Delta$ acol $\Delta$ double mutants lost mtDNA (Figure 6 and data not shown). These data suggest that hydrogen peroxide generated from reactions catalyzed by Sod1 contributes to $\mathrm{mtDNA}$ loss in acol $\Delta$ mutant cells. Mutations in $S O D 1$ also cause oxidative damage due to accumulation of superoxide radicals $[15,16]$. However, since an sod1 $\Delta$ mutation suppressed mtDNA loss in acols mutant cells, we propose that hydroxyl radicals are more damaging to mtDNA than superoxide radicals. How would mutations in the cytosolic isoform of superoxide dismutase rescue a mitochondrial defect? It has been shown that a small fraction of Sod1 is localized in the intermembrane space of mitochondria, which protects cells from mitochondrial 
oxidative damage [45-47]. Why does not sod2 $\Delta$ suppress mtDNA loss associated with acold? It is possible that loss of Sod2 leads to increased levels of superoxide radicals in the mitochondria, which in the presence of ferrous ions would cause any hydrogen peroxide produced in the mitochondria to be converted to the hydroxyl radicals via the Fenton/Haber Weiss reactions that damage mtDNA.

3.7. Loss of mtDNA in aco1 $\Delta$ Mutant Cells Is Growth Medium Dependent. It has been proposed that mtDNA loss in acols mutant cells is due to lack of physical protection of mtDNA by Acol [20]. One key piece of evidence that supports this hypothesis is the observation that the expression of two catalytically inactive Acol mutants, $\mathrm{Acol}^{\mathrm{C} 382 \mathrm{~S}}$ and $\mathrm{Acol}^{\mathrm{C} 445 \mathrm{~S}}$, under the control of the $A D H 1$ promoter from the pRS416 centromeric plasmid, prevented mtDNA loss in acol $\Delta$ mutant cells. To maintain the plasmids, transformants were grown in YNBcasD medium. In light of discovery that iron citrate toxicity contributes to mtDNA loss, one alternative explanation for mtDNA retention in cells expressing $\mathrm{ACOI}^{\mathrm{C} 382 \mathrm{~S}}$ and $A C O 1^{C 445 S}$ mutant alleles is due to differences in growth medium, YNBcasD versus YPD. Accordingly, we transformed aco1 $\triangle / A C O 1$ heterozygous diploid mutant cells with empty pRS416 vector and transformants were sporulated and dissected on YPD or YNBcasD medium. Eleven aco1s haploid mutants from a YPD dissection plate were obtained and grown in YPD liquid medium and mtDNA was observed by DAPI staining. We found that all of the eleven aco1 $\Delta$ segregants lost mitochondrial DNA (Figure 7 and data not shown). In contrast, among nine aco $1 \Delta$ mutant segregants carrying the empty pRS416 vector from a YNBcasD dissection plate that were grown in YNBcasD liquid medium, seven maintained mtDNA (Figure 7 and data not shown). When these seven aco1 $1 \Delta$ mutants containing mtDNA were passed onto YPD plate medium twice and then grown in YPD liquid medium, all lost mtDNA (data not shown). Together, these data suggest that mtDNA loss in acol $\Delta$ mutant cells is growth medium dependent.

\section{Conclusions}

It has been proposed that yeast aconitase (Acol) physically binds to mtDNA and promotes its maintenance [20,21]. Our results in this study suggest a different, but not necessarily mutually exclusive, mechanism. We propose that acol $\Delta$ activates the RTG pathway, resulting in increased citrate production through upregulation of genes encoding citrate synthase. Increased levels of citrate lead to iron overload in the mitochondria. Iron then reacts with hydrogen peroxide to generate hydroxyl radicals, which cause oxidative damage to mitochondrial DNA and consequently its instability. Mutations of yeast frataxin (Yfhl) lead to loss of activity of aconitase [48]. Suppression of mtDNA instability due to mutations in both YFH1 and $A C O 1$ by reduced iron citrate levels raises the possibility that mtDNA loss in $y f h 1$ mutant cells may be an indirect consequence of aconitase inactivation. Our data also suggest that the cytosolic superoxide dismutase, Sod1, but not

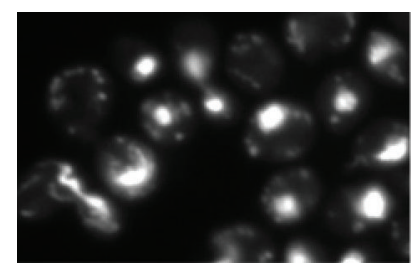

$\operatorname{aco} 1 \Delta(\mathrm{YNBCasD})$

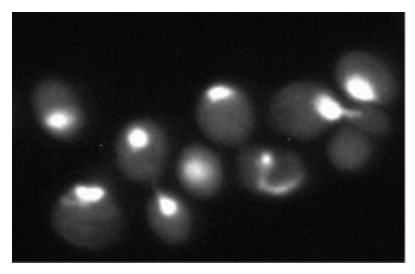

$\operatorname{aco} 1 \Delta(\mathrm{YPD})$
FigURE 7: mtDNA loss due to acol $\Delta$ is growth medium dependent. aco1 $\Delta$ mutant cells (ZLY2630) carrying empty pRS416 vector were grown in YNBcasD or YPD medium to mid-logarithmic phase, stained with DAPI, and examined using fluorescence microscopy.

the mitochondrial superoxide dismutase, Sod2, contributes to mtDNA loss in acol $\Delta$ mutant cells.

\section{Conflict of Interests}

The authors declare no conflict of interests.

\section{Acknowledgments}

The authors would like to thank Dr. Jerry Kaplan at The University of Utah for yeast strains, W. M. Keck Foundation for the Keck Facility, and Robin Rowe for sequencing. This work was supported by grant 1R15GM094772-01A1 (to Z. Liu) from the National Institutes of Health.

\section{References}

[1] F. Foury, T. Roganti, N. Lecrenier, and B. Purnelle, "The complete sequence of the mitochondrial genome of Saccharomyces cerevisiae," FEBS Letters, vol. 440, no. 3, pp. 325-331, 1998.

[2] B. F. Lang, M. W. Gray, and G. Burger, "Mitochondrial genome evolution and the origin of eukaryotes," Annual Review of Genetics, vol. 33, pp. 351-397, 1999.

[3] R. W. Taylor and D. M. Turnbull, "Mitochondrial DNA mutations in human disease," Nature Reviews Genetics, vol. 6, no. 5, pp. 389-402, 2005.

[4] L. C. Greaves, A. K. Reeve, R. W. Taylor, and D. M. Turnbull, "Mitochondrial DNA and disease," Journal of Pathology, vol. 226, no. 2, pp. 274-286, 2012.

[5] A. Siddiqui, S. Rivera-Sanchez, R. Castro Mdel et al., "Mitochondrial DNA damage is associated with reduced mitochondrial bioenergetics in Huntington's disease," Free Radical Biology \& Medicine, vol. 53, no. 7, pp. 1478-1488, 2012.

[6] K. A. Lipinski, A. Kaniak-Golik, and P. Golik, "Maintenance and expression of the S. Cerevisiae mitochondrial genomeFrom genetics to evolution and systems biology," Biochimica et Biophysica Acta, vol. 1797, no. 6-7, pp. 1086-1098, 2010.

[7] V. Contamine and M. Picard, "Maintenance and integrity of the mitochondrial genome: a plethora of nuclear genes in the budding yeast," Microbiology and Molecular Biology Reviews, vol. 64, no. 2, pp. 281-315, 2000.

[8] M. Babcock, D. De Silva, R. Oaks et al., "Regulation of mitochondrial iron accumulation by Yfhlp, a putative homolog of frataxin," Science, vol. 276, no. 5319, pp. 1709-1712, 1997. 
[9] H. Koutnikova, V. Campuzano, F. Foury, P. Dollé, O. Cazzalini, and M. Koenig, "Studies of human, mouse and yeast homologues indicate a mitochondrial function for frataxin," Nature Genetics, vol. 16, no. 4, pp. 345-351, 1997.

[10] R. B. Wilson and D. M. Roof, "Respiratory deficiency due to loss of mitochondrial dna in yeast lacking the frataxin homologue," Nature Genetics, vol. 16, no. 4, pp. 352-357, 1997.

[11] F. Foury and T. Roganti, "Deletion of the mitochondrial carrier genes MRS3 and MRS4 suppresses mitochondrial iron accumulation in a yeast frataxin-deficient strain," Journal of Biological Chemistry, vol. 277, no. 27, pp. 24475-24483, 2002.

[12] O. S. Chen, S. Hemenway, and J. Kaplan, "Genetic analysis of iron citrate toxicity in yeast: implications for mammalian iron homeostasis," Proceedings of the National Academy of Sciences of the United States of America, vol. 99, no. 26, pp. 16922-16927, 2002.

[13] V. C. Culotta, "Superoxide dismutase, oxidative stress, and cell metabolism," Current Topics in Cellular Regulation C, vol. 36, pp. 117-132, 2001.

[14] C. M. Grant, G. Perrone, and I. W. Dawes, "Glutathione and catalase provide overlapping defenses for protection against hydrogen peroxide in the yeast Saccharomyces cerevisiae," Biochemical and Biophysical Research Communications, vol. 253, no. 3, pp. 893-898, 1998.

[15] O. Bermingham-McDonogh, E. B. Gralla, and J. S. Valentine, "The copper, zinc-superoxide dismutase gene of Saccharomyces cerevisiae: cloning, sequencing, and biological activity," Proceedings of the National Academy of Sciences of the United States of America, vol. 85, no. 13, pp. 4789-4793, 1988.

[16] X. F. Liu, I. Elashvili, E. B. Gralla, J. S. Valentine, P. Lapinskas, and V. C. Culotta, "Yeast lacking superoxide dismutase. Isolation of genetic suppressors," Journal of Biological Chemistry, vol. 267, no. 26, pp. 18298-18302, 1992.

[17] A. Lin, K. W. Hakala, S. T. Weintraub, and L. McAlister-Henn, "Suppression of metabolic defects of yeast isocitrate dehydrogenase and aconitase mutants by loss of citrate synthase," Archives of Biochemistry and Biophysics, vol. 474, no. 1, pp. 205-212, 2008.

[18] M. T. McCammon, C. B. Epstein, B. Przybyla-Zawislak, L. McAlister-Henn, and R. A. Butow, "Global transcription analysis of Krebs tricarboxylic acid cycle mutants reveals an alternating pattern of gene expression and effects on hypoxic and oxidative genes," Molecular Biology of the Cell, vol. 14, no. 3, pp. 958-972, 2003.

[19] M. Merkel and J. Kretowicz, "Sensitivity to natamycin in vitro evaluation of Candida spp. and Torulopsis glabrata isolated from vagina," Polski Tygodnik Lekarski, vol. 40, no. 9, pp. 257258,1985 .

[20] X. J. Chen, X. Wang, and R. A. Butow, "Yeast aconitase binds and provides metabolically coupled protection to mitochondrial DNA," Proceedings of the National Academy of Sciences of the United States of America, vol. 104, no. 34, pp. 13738-13743, 2007.

[21] X. J. Chen, X. Wang, B. A. Kaufman, and R. A. Butow, "Aconitase couples metabolic regulation to mitochondrial DNA maintenance," Science, vol. 307, no. 5710, pp. 714-717, 2005.

[22] Z. Liu and R. A. Butow, "A transcriptional switch in the expression of yeast tricarboxylic acid cycle genes in response to a reduction or loss of respiratory function," Molecular and Cellular Biology, vol. 19, no. 10, pp. 6720-6728, 1999.

[23] Z. Liu and R. A. Butow, "Mitochondrial retrograde signaling," Annual Review of Genetics, vol. 40, pp. 159-185, 2006.
[24] Z. Liu, T. Sekito, M. Špírek, J. Thornton, and R. A. Butow, "Retrograde signaling is regulated by the dynamic interaction between Rtg2p and Mkslp," Molecular Cell, vol. 12, no. 2, pp. 401411, 2003

[25] S. P. Gangloff, D. Marguet, and G. J.-M. Lauquin, "Molecular cloning of the yeast mitochondrial aconitase gene (ACO1) and evidence of a synergistic regulation of expression by glucose plus glutamate," Molecular and Cellular Biology, vol. 10, no. 7, pp. 3551-3561, 1990.

[26] M. T. McCammon, "Mutants of Saccharomyces cerevisiae with defects in acetate metabolism: isolation and characterization of Acn- mutants," Genetics, vol. 144, no. 1, pp. 57-69, 1996.

[27] C. Vélot, P. Haviernik, and G. J.-M. Lauquin, "The Saccharomyces cerevisiae RTG2 gene is a regulator of aconitase expression under catabolite repression conditions," Genetics, vol. 144, no. 3, pp. 893-903, 1996.

[28] D. C. Amberg, D. J. Burke, and J. N. Strathern, Methods in Yeast Genetics: A Cold Spring Harbor Laboratory Course Manual, Cold Spring Harbor Laboratory, Cold Spring Harbor, NY, USA, 2005.

[29] L. Li and J. Kaplan, "A mitochondrial-vacuolar signaling pathway in yeast that affects iron and copper metabolism," Journal of Biological Chemistry, vol. 279, no. 32, pp. 33653-33661, 2004.

[30] R. S. Sikorski and P. Hieter, "A system of shuttle vectors and yeast host strains designed for efficient manipulation of DNA in Saccharomyces cerevisiae," Genetics, vol. 122, no. 1, pp. 19-27, 1989.

[31] B. A. Rothermel, A. W. Shyjan, J. L. Etheredge, and R. A. Butow, "Transactivation by Rtglp, a basic helix-loop-helix protein that functions in communication between mitochondria and the nucleus in yeast," Journal of Biological Chemistry, vol. 270, no. 49, pp. 29476-29482, 1995.

[32] Y. Jia, B. Rothermel, J. Thornton, and R. A. Butow, "A basic helixloop-helix-leucine zipper transcription complex in yeast functions in a signaling pathway from mitochondria to the nucleus," Molecular and Cellular Biology, vol. 17, no. 3, pp. 1110-1117, 1997.

[33] A. Chelstowska, Z. Liu, Y. Jia, D. Amberg, and R. A. Butow, "Signalling between mitochondria and the nucleus regulates the expression of a new D-lactate dehydrogenase activity in yeast," Yeast, vol. 15, no. 13, pp. 1377-1391, 1999.

[34] Z. Liu, T. Sekito, C. B. Epstein, and R. A. Butow, "RTGdependent mitochondria to nucleus signaling is negatively regulated by the seven WD-repeat protein Lst8p," EMBO Journal, vol. 20, no. 24, pp. 7209-7219, 2002.

[35] T. Sekito, Z. Liu, J. Thornton, and R. A. Butow, "RTG-dependent mitochondria-to-nucleus signaling is regulated by MKS1 and is linked to formation of yeast prion [URE3]," Molecular Biology of the Cell, vol. 13, no. 3, pp. 795-804, 2002.

[36] X. Liao, W. C. Small, P. A. Srere, and R. A. Butow, "Intramitochondrial functions regulate nonmitochondrial citrate synthase (CIT2) expression in Saccharomyces cerevisiae," Molecular and Cellular Biology, vol. 11, no. 1, pp. 38-46, 1991.

[37] P. A. Kirchman, S. Kim, C. Lai, and S. Michal Jazwinski, "Interorganelle signaling is a determinant of longevity in Saccharomyces cerevisiae," Genetics, vol. 152, no. 1, pp. 179-190, 1999.

[38] D. H. Williamson and D. J. Fennell, "Visualization of yeast mitochondrial dna with the fluorescent stain 'DAPI"' Methods in Enzymology C, vol. 56, pp. 728-733, 1979.

[39] Y.-K. Jia, A.-M. Bécam, and C. J. Herbert, “The CIT3 gene of Saccharomyces cerevisiae encodes a second mitochondrial isoform of citrate synthase," Molecular Microbiology, vol. 24, no. 1, pp. 53-59, 1997. 
[40] K. S. Kim, M. S. Rosenkrantz, and L. Guarente, "Saccharomyces cerevisiae contains two functional citrate synthase genes," Molecular and Cellular Biology, vol. 6, no. 6, pp. 1936-1942, 1986.

[41] A. S. Lewin, V. Hines, and G. M. Small, "Citrate synthase encoded by the CIT2 gene of Saccharomyces cerevisiae is peroxisomal," Molecular and Cellular Biology, vol. 10, no. 4, pp. 1399-1405, 1990.

[42] U. Mühlenhoff, J. A. Stadler, N. Richhardt et al., "A specific role of the yeast mitochondrial carriers Mrs3/4p in mitochondrial iron acquisition under iron-limiting conditions," Journal of Biological Chemistry, vol. 278, no. 42, pp. 40612-40620, 2003.

[43] E. M. Froschauer, R. J. Schweyen, and G. Wiesenberger, "The yeast mitochondrial carrier proteins Mrs3p/Mrs4p mediate iron transport across the inner mitochondrial membrane," Biochimica et Biophysica Acta, vol. 1788, no. 5, pp. 1044-1050, 2009.

[44] J. C. Rutherford and A. J. Bird, "Metal-responsive transcription factors that regulate iron, zinc, and copper homeostasis in eukaryotic cells," Eukaryotic Cell, vol. 3, no. 1, pp. 1-13, 2004.

[45] H. Kawamata and G. Manfredi, "Import, maturation, and function of SOD1 and its copper chaperone CCS in the mitochondrial intermembrane space," Antioxidants and Redox Signaling, vol. 13, no. 9, pp. 1375-1384, 2010.

[46] C. Klöppel, C. Michels, J. Zimmer, J. M. Herrmann, and J. Riemer, "In yeast redistribution of Sod1 to the mitochondrial intermembrane space provides protection against respiration derived oxidative stress," Biochemical and Biophysical Research Communications, vol. 403, no. 1, pp. 114-119, 2010.

[47] L. A. Sturtz, K. Diekert, L. T. Jensen, R. Lill, and V. C. Culotta, "A fraction of yeast $\mathrm{Cu}, \mathrm{Zn}$-superoxide dismutase and its metallochaperone, CCS, localize to the intermembrane space of mitochondria. A physiological role for SOD1 in guarding against mitochondrial oxidative damage," Journal of Biological Chemistry, vol. 276, no. 41, pp. 38084-38089, 2001.

[48] F. Foury, "Low iron concentration and aconitase deficiency in a yeast frataxin homologue deficient strain," FEBS Letters, vol. 456, no. 2, pp. 281-284, 1999. 


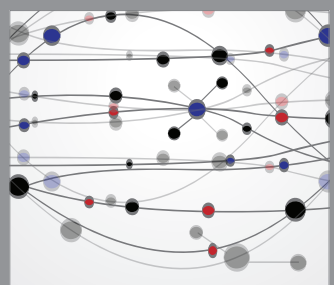

The Scientific World Journal
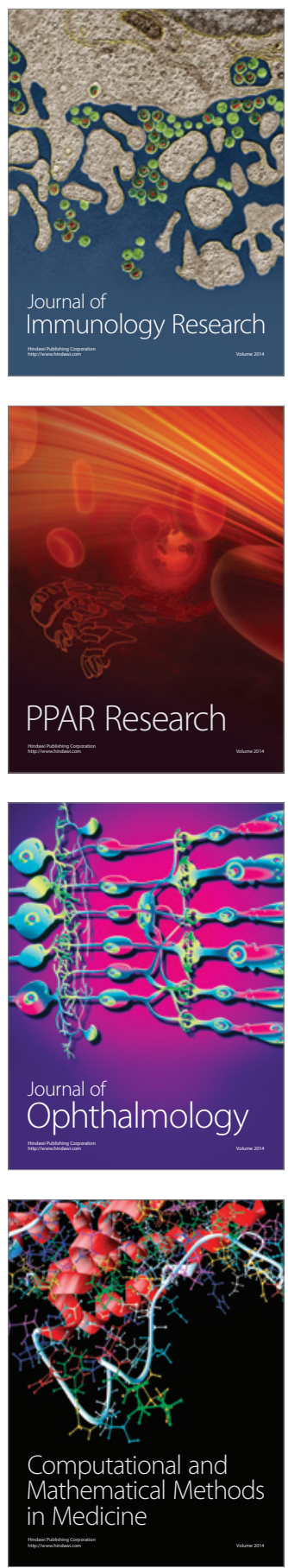

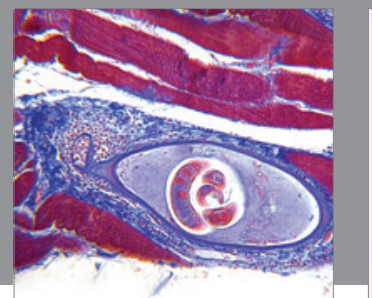

Gastroenterology

Research and Practice
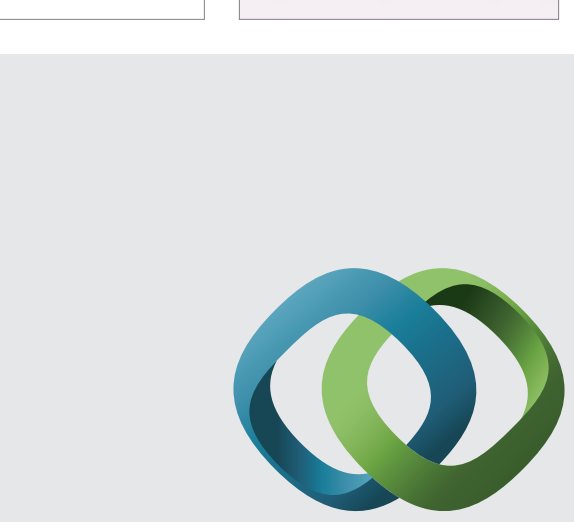

\section{Hindawi}

Submit your manuscripts at

http://www.hindawi.com
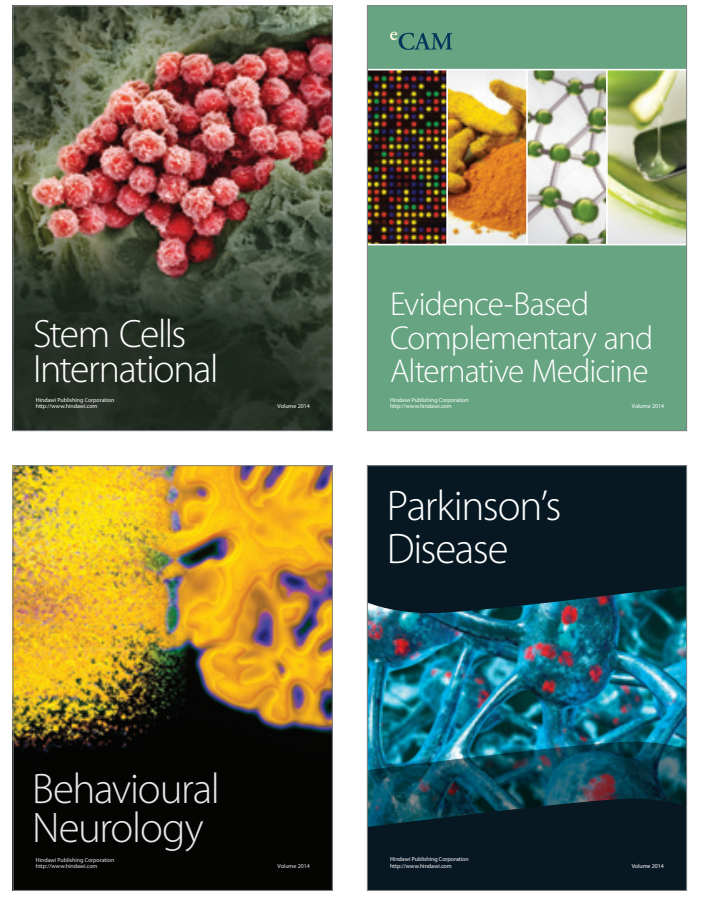
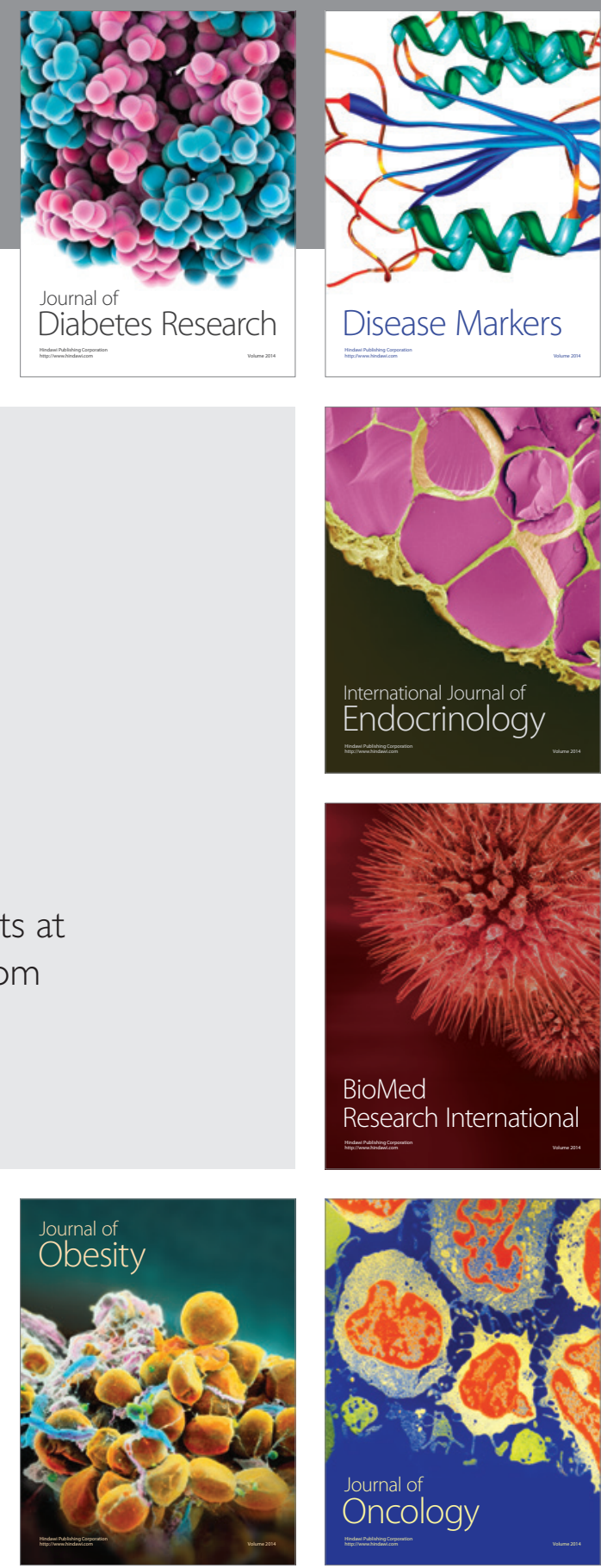

Disease Markers
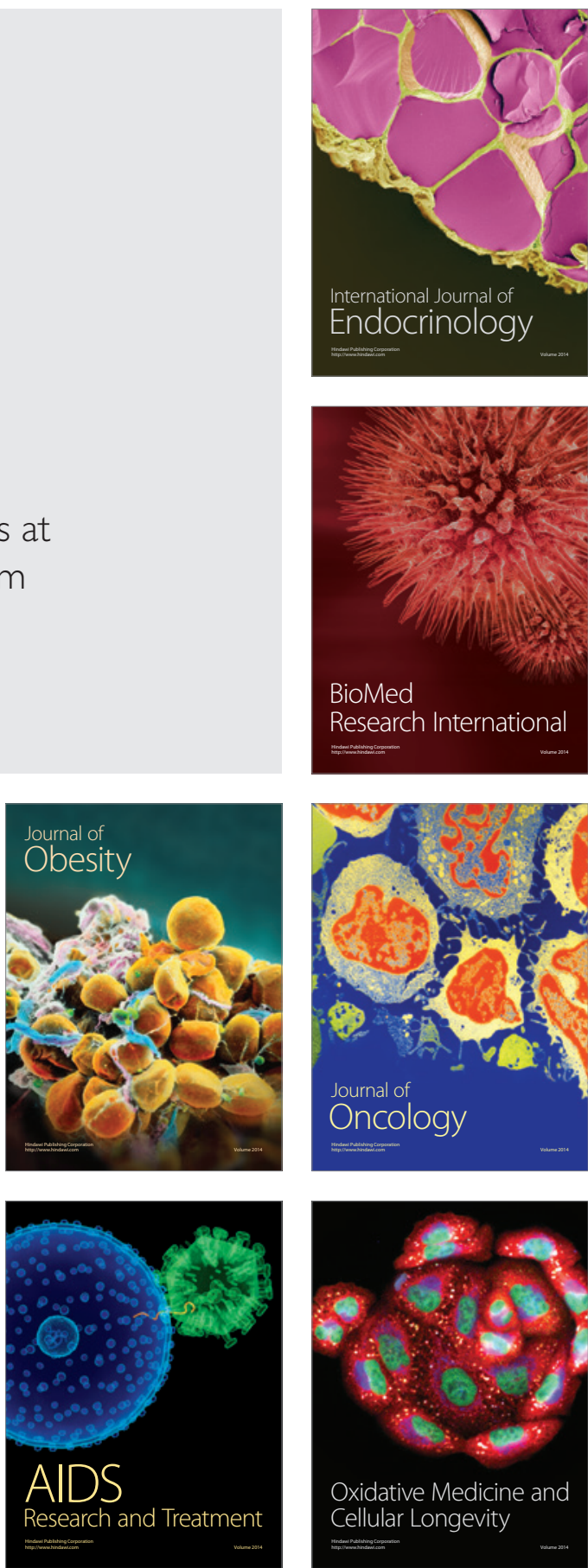